\title{
Frontières
}

\section{Comme une odeur de chair fraîche}

\section{L'ogresse dans trois romans contemporains}

\section{Patrick Bergeron}

Volume 23, numéro 2, printemps 2011

Enquêtes sur le cadavre : 2. Fantastique

URI : https://id.erudit.org/iderudit/1007584ar

DOI : https://doi.org/10.7202/1007584ar

Aller au sommaire du numéro

Éditeur(s)

Université du Québec à Montréal

ISSN

1916-0976 (numérique)

Découvrir la revue

Citer cet article

Bergeron, P. (2011). Comme une odeur de chair fraîche : l'ogresse dans trois romans contemporains. Frontières, 23(2), 14-20.

https://doi.org/10.7202/1007584ar
Résumé de l'article

Cet article s'inspire de la réapparition de la figure de l'ogre dans le roman contemporain d'expression française (Tournier, Chessex, Pennac) pour examiner le rôle dévolu à l'ogresse. Complice et alliée traditionnelle de l'ogre, celle-ci donne lieu, chez Paul Morand, Gabrielle Wittkop et Dynah Psyché, à une caractérisation amorale et ambiguë qui soulève la question : quelle est la valeur esthétique de l'abjection? d'utilisation que vous pouvez consulter en ligne.

https://apropos.erudit.org/fr/usagers/politique-dutilisation/ 


\section{Résumé}

Cet article s'inspire de la réapparition de la figure de l'ogre dans le roman contemporain d'expression française (Tournier, Chessex, Pennac) pour examiner le rôle dévolu à l'ogresse. Complice et alliée traditionnelle de l'ogre, celle-ci donne lieu, chez Paul Morand, Gabrielle Wittkop et Dynah Psyché, à une caractérisation amorale et ambiguë qui soulève la question: quelle est la valeur esthétique de l'abjection?

Mots clés: ogres et ogressescannibalisme-mort et sexualitémal infligé aux enfants.

\section{Abstract}

Inspired by the reappearance of the ogre in the works of modern French novelists (Tournier, Chessex, Pennac), this article examines the role of his traditional ally and counterpart: the ogress. Amorally and ambiguously depicted by Paul Morand, Gabrielle Wittkop and Dynah Psyché, the ogress brings us to question the aesthetic relevance of abjection.

Keywords: ogres and ogressescannibalism-death and sexualityharm inflicted on children.

\section{COMME UNE ODEUR DE CHAIR FRAÎCHE L'ogresse dans trois romans contemporains ${ }^{1}$}

\author{
POUR LA PLUPART NOUS PRÉFÉRONS LA CHAIR BIEN FRAîCHE \\ EN PARTICULIER CELLE DES PETITS ENFANTS \\ LA VIE QUI CROQUE SOUS LA DENT \\ ET QUI GÉMIT ENCORE LONGTEMPS DANS L'ESTOMAC \\ (MICHEL BUTOR, «DANSE DES OGRES », KRELL, 2009, P. 124)
}

Patrick Bergeron, Ph.D.,

professeur adjoint, Département d'études françaises, Université du Nouveau-Brunswick.

L'ogre, cet affreux géant dévorateur d'enfants auquel nous ont habitués les contes de fées, a inspiré divers romanciers français depuis quatre décennies. L'universitaire américain Jonathan $\mathrm{F}$. Krell affirme qu'on assiste à rien de moins qu'une renaissance de cette figure mythique apparue au Moyen Âge. Or, les œuvres que considère Krell ${ }^{2}$ - notamment Le roi des aulnes de Michel Tournier, L'ogre de Jacques Chessex et $A u$ bonheur des ogres de Daniel Pennac-ne relèvent pas à proprement parler de la littérature enfantine. C'est l'un des premiers paradoxes que signale Krell. Mais ce n'est pas le seul. Autre chose a changé depuis les récits de Perrault ${ }^{3}$. Dans des contes comme «Le petit Poucet» ou «Le chat botté», l'intrigue suivait un canevas somme toute optimiste: les enfants parvenaient à déjouer la vigilance de l'ogre et à échapper à sa voracité destructrice. On voyait se réaffirmer un triomphe de petits David courageux contre d'ignobles Goliath cannibales. Ce scénario favorable aux enfants donnait l'indication d'une société prospère où les plus âgés cédaient leur place aux plus jeunes. Il s'agissait, en fin de compte, d'un univers où s'élaborait une vision de l'avenir.

Chez les romanciers contemporains, l'ogre donne lieu à des intrigues pour le moins ambiguës. Il s'agit d'un mythe «extraordinairement sombre» (overwhelmingly bleak) affirme Krell, car c'est l'ogre qui tend désormais à l'emporter sur les enfants. La vision de la société, en arrièreplan, revêt du même coup un air maussade, voire malsain. Nouveau paradoxe: cette vision assombrie entre pour beaucoup dans le regain d'intérêt dont bénéficie le mythe de l'ogre. Pour Krell, cela s'explique par notre fascination pour la nature ambiguë de l'être humain: "[...] I conclude that the ogre metaphor is so compelling because ogres are a part of us as much as they are the monstrous Other. Like the Titan Cronus, they incarnate the ambiguity of humanity, capable of both immense good and immeasurable evil» (Krell, 
N’EST-CE PAS ÉTONNANT QUE L'OGRE TENDE

À CE POINT À ÉCLIPSER L'OGRESSE ?

POURTANT, DÉJÀ CHEZ PERRAULT, LA FIGURE DE L'OGRESSE,

QUOIQUE SUBALTERNE, PRÉSENTAIT UN RÉEL INTÉRÊT.

2009, p. 22) Cette aptitude à la «bonté immense» et au «mal incommensurable» justifie une bonne part de l'intérêt qu'a pu porter Michel Tournier, l'auteur du Roi des aulnes, à la figure de l'ogre historique Gilles de Rais (1404-1440), compagnon d'armes de Jeanne d'Arc devenu sadique massacreur de jeunes garçons.

Qu'est-ce qui empêche le monstre tapi en nous de donner libre cours à son ignominie? On s'est beaucoup posé la question dans l'ère moderne, surtout depuis l'avènement de l'Allemagne nazie.

Un aspect surprend sitôt qu'il est question du mythe de l'ogre: son caractère foncièrement masculin. Même le dictionnaire consigne cette inégalité. Le Petit Robert révèle que le mot ogre a fait son entrée dans la langue française vers 1300, alors que son pendant féminin, ogresse, n'est attesté qu'à compter de 1697 - l'année où Perrault publie les Contes de ma mère l'oye. Dans les romans déjà évoqués de Tournier et de Chessex, les ogres représentés sont des hommes. Pascal Bruckner, dans son récit Les ogres anonymes, relate l'histoire d'un ogre, Balthus Zaminski, qui rencontre certes une ogresse, Marylène; mais cette séquence n'occupe qu'une mince part du récit.

N'est-ce pas étonnant que l'ogre tende à ce point à éclipser l'ogresse ? Pourtant, déjà chez Perrault, la figure de l'ogresse, quoique subalterne, présentait un réel intérêt.

L'écrivaine Pierrette Fleutiaux, intriguée par l'ogresse, a voulu savoir ce qui fait la valeur de cette figure prise isolément. «Dans Le Petit Poucet, explique-t-elle, le personnage qui me poursuivait, c'est la femme de l'Ogre, qui n'apparaît que dans quelques lignes, qui est à la fois si effacée et si hardie, et dont la situation est si étrange puisque c'est par elle que viennent en contact le monde le plus cruel et le monde le plus innocent » (Fleutiaux, 2010, p. 11). Dans Métamorphoses de la reine, on trouve un conte intitulé "La femme de l'Ogre», qui se veut une réécriture du «Petit Poucet». Fleutiaux y dépeint son héroïne "végétarienne, et bien affligée de ses sept petites ogrelettes aux quenottes trop avides et de cet époux tourmenté par un affreux instinct de mort» (Fleutiaux, 2010, 4e de couverture). On l'aura noté: le statut d'ogre et le végétarisme sont diamétralement opposés. Il y aurait beaucoup à dire à propos de ce conte, qui a inspiré un opéra à Monic Cecconi-Botella. Je note seulement, pour les besoins de mon propos, que le récit de Fleutiaux profite de l'ambiguïté du mythe. «La femme de l'Ogre, lit-on dès l'incipit, n'aime pas préparer la chair, mais elle ne le sait pas» (Fleutiaux, 2010, p. 15).

Ainsi qu'on l'a vu, l'ogresse était déjà chez Perrault une figure ambiguë. D'une part, c'est elle qui aide le Petit Poucet et ses frères en substituant les couronnes d'or. C'est aussi elle, d'autre part, qui permet que ses sept filles soient égorgées. On a déjà vu mère plus attentionnée.

Les filles de l'ogre appellent à leur tour quelques observations. Dans «La femme de l'Ogre », Fleutiaux leur donne moins de relief qu'à l'héroïne éponyme. Pourtant, Perrault en faisait une description, certes laconique, mais suffisante pour glacer le sang:

Ces petites Ogresses avaient toutes le teint fort beau, parce qu'elles mangeaient de la chair fraîche comme leur père; mais elles avaient de petits yeux gris et tout ronds, le nez crochu et une fort grande bouche avec de longues dents fort aiguës et fort éloignées l'une de l'autre. Elles n'étaient pas encore fort méchantes; mais elles promettaient beaucoup, car elles mordaient déjà les petits enfants pour en sucer le sang (Perrault, 2004, p. 196).

Bien sûr, Perrault les fait mourir. La substitution des couronnes d'or permet au Petit Poucet et à ses frères de survivre. L'ogre, persuadé que les petites silhouettes endormies sont celles de jeunes intrus dont la chair fraîche l'affriande, ignore totalement qu'il s'agit en réalité de ses filles et il ne s'en rend compte que trop tard, après les avoir dévorées. Pourtant, la vocation malveillante de ces jeunes victimes (qui n'éveillent guère de compassion) ne nous aura pas échappé: il s'agissait, avant leur mort, de monstres en devenir; «elles promettaient beaucoup », indiquait Perrault.

Comme pour Fleutiaux, c'est la figure de l'ogresse qui m'intéresse avant tout ici. J'aimerais montrer dans quelle mesure celle-ci peut donner lieu à des traitements ambigus. Alliée traditionnelle de l'ogre dont elle est généralement l'épouse (ou la fille), l'ogresse tient un rôle encore plus vague. La verra-t-on apparaître seule, sans affiliation avec un ogre? Certains lecteurs, férus d'affaires criminelles, se souviendront de Jeanne Weber (1874-1910), surnommée «l'Ogresse de la Goutte d'or » et reconnue coupable de dix meurtres d'enfants par étouffement. Contrairement à d'autres faits divers ${ }^{4}$, celui-ci ne semble guère avoir inspiré les auteurs de fiction.

En littérature, voit-on souvent l'ogresse tenir le premier rôle? Il appert que non, jusqu'ici du moins, car tout pourrait changer.

Trois œuvres ont retenu mon attention. Elles ne font peut-être pas partie du corpus de Jonathan F. Krell et ne font peut-être pas explicitement référence, pour deux d'entres elles, au mythe de l'ogre. Cependant, elles n'en proposent pas moins des variations significatives et pour le moins subversives. J'aimerais montrer que les romans Hécate et ses chiens (1954) de Paul Morand, La marchande d'enfants (2003) de Gabrielle Wittkop et L'ogresse (2011) de Dynah Psyché représentent des cas fascinants quoique dérangeants de ce que peut la littérature au regard de la monstruosité et de l'abjection. Puisque l'horreur remplit ici un tout autre rôle qu'elle ne le fait, par exemple, dans la littérature de la Shoah (où l'horreur permet soit de témoigner de ce dont l'homme est capable, soit de remplir un devoir de mémoire envers les victimes), il me paraît important de nous interroger: pourquoi écrire de tels textes? Et $a$ fortiori: pourquoi les lire? Jusqu'où un auteur peut-il aller? Nous verrons que Morand, Wittkop et Psyché abordent ces problèmes de trois manières différentes.

À une époque comme la nôtre, où la violence, voire l'ultra-violence ${ }^{5}$, imprègne de plus en plus fortement la culture populaire, nous nous sommes défaits de plusieurs tabous en matière de sexualité et de mort. Or, le meurtre et la torture d'enfants relèvent toujours de l'insoutenable. Au cinéma par exemple, il est rare qu'on montre des cadavres d'enfants, même dans les films d'épouvante. S'il veut le faire, un cinéaste devra user d'un doigté que n'exigent pas les cadavres d'adultes ou de masse ${ }^{6}$.

Je propose de lire Hécate et ses chiens, La marchande d'enfants et L'ogresse à la lumière d'un changement de fonction narrative du monstre, un changement survenu-si l'on en croit Krell-depuis quelques décennies (depuis l'après-guerre, disons). Traditionnellement, à la suite de Perrault, les ogres désignent des éléments perturbateurs majeurs dont les héros sont appelés à triompher. De nos jours, ogres et ogresses participent à un souci d'ambiguïté narrative, qui s'est considérablement 
amplifié depuis que les romans de serialkillers (pensons aux œuvres de Jim Thompson, Shane Stevens, Thomas Harris ou Jeff Lindsay) sont en vogue. Qu'ils aient nom Lou Ford, Thomas Bishop, Hannibal Lecter ou Dexter Morgan, les meurtriers ont la cote. Voilà qui suffit pour offrir aux ogres et aux ogresses un horizon d'accueil des plus favorables.

\section{DES FRUITS AMERS \\ ET SANS DANGER?}

Comme on l'a vu, ce qui frappe de prime abord dans le contexte contemporain, c'est que l'ogre, tout comme sa contrepartie féminine, l'ogresse, sont l'incarnation d'un processus de subversion mythologique. Dans les contes traditionnels, tels qu'on les connaît depuis Perrault et Mme d'Aulnoy, l'ogre déployait un appétit pour la chair fraîche et terrifiait les enfants. Ceux-ci, en revanche, se voyaient offrir un scénario de victoire.

Il en va tout autrement de nos jours. Subverti, le mythe privilégie désormais le triomphe de l'ogre et sous-tend, de la part des écrivains, une fascination pour les figures de criminels sadiques, voire cannibales. Cette "inversion maligne», pour reprendre l'expression consacrée de Michel Tournier, en dit long, à mon avis, sur l'avidité avec laquelle notre époque se délecte de mythes violents et transgressifs, surtout depuis les années 1960 et 1970, où les apports cumulatifs du cinéma, des séries télévisées et, plus récemment, des jeux vidéo, a sans cesse étendu le domaine de l'horreur. L'ogre, au fil de ses incarnations, fussent-elles de nature politique, sexuelle ou familiale, nous permet peut-être de toucher le fond de cette "approche de l'abjection", pour emprunter une expression, cette fois, à Julia Kristeva ${ }^{7}$. Dans la littérature enfantine traditionnelle, l'abjection qui émane de l'ogre est adoucie. À preuve, cette affirmation tirée du « Petit Poucet»: «cet Ogre ne laissait pas d'être un fort bon mari, quoiqu'il mangeât les petits enfants» (Perrault, 2004, p. 199). Il ne faut pas prendre cette proposition concessive à la légère: tout bon mari que soit l'ogre, la dévoration, la digestion et l'anthropophagie sont les principaux aspects de son mythe.

L'ogre n'est pas un simple cannibale, car il se délecte de chair d'enfants. Sur ce plan, de multiples exploitations sont possibles, de nombreuses interprétations sont permises, car il existe divers degrés à l'horreur. Ainsi pour Tournier, le comble de la terreur est atteint dans l'«infantiphagie ${ }^{8}$ », cette pratique cannibale consistant à se repaître de la chair de ses propres enfants. Dynah Psyché y fait allusion ${ }^{9}$.
Dans l'optique que je privilégie ici, le mal fait aux enfants, voire l'exploitation sexuelle des mineurs, nous avançons aux limites du supportable, surtout dans le contexte de l'après-affaire Dutroux et d'autres enquêtes judiciaires lourdement médiatisées qui en ont fait des sujets sensibles. De pédophilie, il est assurément question à travers les deux premières figures d'ogresses sur lesquelles je me suis penché: le personnage de Clotilde, chez Morand, est une Européenne trentenaire dans l'Afrique du Nord des années 1920, qui se met en chasse, la nuit venue, de "petits corps offerts à sa convoitise» (Morand, 1984, p. 121). Le personnage de Marguerite Paradis, chez Gabrielle Wittkop, est une maquerelle pratiquant le scandaleux commerce des enfants perdus

\section{EN LITTÉRATURE,}

VOIT-ON SOUVENT

\section{L'OGRESSE TENIR LE PREMIER}

\section{RÔLE ? IL APPERT QUE NON,}

JUSQU'ICI DU MOINS,

\section{CAR TOUT POURRAIT CHANGER.}

dans le Paris révolutionnaire. Morand use d'un art de la suggestion et de l'ellipse pour suivre le filon de l'obsession amoureuse. Wittkop recourt à une écriture orfévrée, quoique souvent très crue, pour narrer les aléas d'un marchandage obscène et criminel. Dans ce dernier cas, le livre contient non seulement des scénarios outrageux d'agressions sexuelles contre les enfants; il manifeste également une pédophobie d'une rare virulence ${ }^{10}$.

Dans Hécate et ses chiens, l'ogresse, Clotilde, est placée sous le signe d'une divinité antique, malveillante et ambiguë: Hécate. La superposition du mythe moderne (l'ogre) et d'un mythe ancien a déjà suscité d'autres rapprochements, notamment avec Saturne-Cronos (dont la «peinture noire» de Goya est certainement l'une des représentations les plus illustres), mais jamais, à ma connaissance, avec Hécate ${ }^{11}$. Le texte de Morand constitue donc un cas inusité de réécriture mythologique. La narration est assumée par l'amant de Clotilde, un dénommé Spitzgartner. Ce représentant de banque, huguenot «de naissance, de tempérament et de formation» (Morand, 1984, p. 43), développe une fascination, malgré lui (du moins, c'est ce qu'il prétend), pour la double vie nocturne de sa mystérieuse maîtresse. Car mystère il y a: Morand instaure dans son texte un persistant climat d'irrésolution. Clotilde s'est-elle réellement livrée aux débauches répréhensibles que rapporte le narrateur ou celui-ci a-t-il seulement projeté sur elle une déviance qui ne concerne que lui? Morand reste élusif. Au lecteur de trancher. Le récit se compose de soixante-sept chapitres de longueur variable (les plus longs font quelques pages, le plus court tient en une seule ligne). Morand avait 66 ans au moment de sa publication. En juillet 1953, le décret de révocation dont il avait été l'objet en 1944 (l'écrivain-diplomate avait été ambassadeur pour le gouvernement de Vichy) venait d'être annulé. Qu'importe: Morand, s'il avait été célébré dans l'entredeux-guerres, avait désormais mauvaise réputation.

Pour sa part, le roman épistolaire posthume de Gabrielle Wittkop, La marchande d'enfants (2003), relate le scandaleux commerce des enfants perdus au XVIII ${ }^{\mathrm{e}}$ siècle, du point de vue de l'ogresse (la maquerelle Marguerite Paradis). Cette fois, nulle déité ancienne n'est invoquée autour de l'ogre. C'est plutôt à l'enseigne du marquis de Sade - «Donatien l'Admirable», à qui le livre est dédié-que Wittkop explore, à son tour, l'ambigu, voire repoussant filon de la perversion des désirs amoureux. Le choix du XVIII ${ }^{\mathrm{e}}$ siècle n'a rien de surprenant pour quiconque a lu d'autres textes de Wittkop. «Outre le marquis de Sade, explique Jérôme Garcin dans Les livres ont un visage, elle se réclame de Voltaire, La Mettrie, Holbach, Condillac, d'Alembert, Diderot, et prétend descendre, en ligne directe, des Lumières » (Garcin, 2009, p. 38). La narration se déroule entre le 27 mai 1789 et le 7 germinal de l'an IV (c'est-à-dire le 27 mars 1796) ${ }^{12}$. Elle se divise en 27 lettres. Les 26 premières sont datées de Paris et adressées par Marguerite à son amie Louise, qui veut ouvrir à Bordeaux une maison de prostitution juvénile comme celle de la tenancière parisienne. La dernière lettre, datée de Bordeaux, est adressée par Louise L. au Citoyen R.

Quoique moins connue que Morand («Les dictionnaires de littérature l'ignorent», observe Garcin [2009, p. 44]), Wittkop a elle aussi une réputation douteuse, mais pour de tout autres motifs. Elle est attribuable en grande partie à son roman Le Nécrophile (1972). Paru chez Régine Desforges Éditeur à la collection «Bibliothèque noire », ce texte, qui fit scandale, explore, sur le mode d'un récit au «je », un des plus tenaces tabous en matière de sexualité marginale, la nécrophilie. L'inspiration sadienne est indéniable. Dans La marchande d'enfants, la «vieille 
dame indigne», ainsi que la surnomme Jérôme Garcin, s'y commet de nouveau: «Des amis à qui j'ai prêté le manuscrit me disent que je vais encore plus loin que Sade dans Les Cent Vingt Journées de Sodome» (Garcin, 2009, p. 44). Affirmer que les livres de Gabrielle Wittkop ne sont pas à mettre entre toutes les mains tient de l'euphémisme. Conscient du terrain miné sur lequel la romancière s'est aventurée, Nikola Delescluse ouvre sa préface intitulée «En guise de paratonnerre» en citant Lautréamont: "Il n'est pas bon que tout le monde lise les pages qui vont suivre: quelques-uns seuls savoureront ce fruit sans danger ${ }^{13}$.» (Wittkop, 2003, p. 9).

Entre le récit elliptique de Morand et le sulfureux roman posthume de Wittkop, L'ogresse de Dynah Psyché semble occuper un juste milieu. Je dis bien «semble», car, on le verra, rien n'est moins sûr. Alors que les deux auteurs français usent d'une prose ancrée, par sa mesure pour l'un et par sa hardiesse pour l'autre, dans le XVIII ${ }^{\mathrm{e}}$ siècle, Psyché (auteure québécoise d'origine martiniquaise) recourt à une langue aux couleurs créoles ${ }^{14}$. Comme l'ont fait Morand et Wittkop avant elle, l'auteure opte pour le récit personnel. C'est l'ogresse, une dénommée Sophonie, qui assure la narration. Elle relate comment elle a «hérité de l'ogritude » (Psyché, 2011, p. 16) - ce qu'elle appelle également «le don»-et comment elle a appris à le servir, car «[l]e don est le grand maître. C'est lui qui pense et décide» (p. 19). Ce don s'est d'abord manifesté par une «capacité phénoménale d'absorption» (p. 41). Enfant, la narratrice dévorait de tout, littéralement:

Je n'ai jamais rien refusé. Les mangots, les dachines, les avocats, les tinains, la morue les jours gras, les ignames avec de l'huile les jours maigres. J'y ai ajouté, de mon propre chef, les cacas nez (sucrés...), le caca zoreilles (amer...), le croissant noir, parfois sableux, des rognures d'ongles, les pépites tarabiscotées des cacas zyeux, etc. Tout ce que produisait mon corps était comestible. C'est toujours vrai (p. 18).

Puis, passé l'âge de douze ans, ses goûts se précisent. La narratrice adopte graduellement une diète transgressive. Elle commence par déguster le petit doigt du bébé Rélucien, sectionné accidentellement. Cette expérience la révèle à elle-même. Elle engloutira ensuite des placentas de nouveau-nés et des fœtus. Elle ne tue pas, ou du moins, pas de gaîté de cœur:

Ce que j'aime, maintenant, c'est cuisiner. Pas tuer. J'éprouve un profond respect pour la vie sous toutes ses formes.
Le malheur veut qu'il faille tuer pour se nourrir. Ce monde est imparfait. Mais ce n'est pas moi qui l'ai créé.

La tuerie m'ennuie, quand il y a

du jus. Ça crie et ça salit. Il y en a

partout, quand le manger se débat

(Psyché, 2011, p. 93).

On le voit, ce ne sont pas des scrupules moraux qui guident l'ogresse, mais des motifs hygiéniques. On comprend alors que l'étouffement puisse passer, aux yeux de la narratrice, pour la meilleure méthode de mise à mort: "C'est propre et net. / Ensuite, pour découper, je suis tranquille» (p. 93). Un boucher ne parlerait pas autrement d'un lapin.

Or, Psyché n'a pas voulu faire le portrait d'une criminelle mais, littéralement, d'une «ogresse». Des trois œuvres que j'examine, c'est ce texte qui file avec le plus d'attention la métaphore de l'appétit démesuré - «Pantagruélique» (p. 87), dira la narratrice.

Par deux traits ${ }^{15}$, L'ogresse se rapproche davantage d'Hécate et ses chiens: la narration rétrospective et le format des chapitres. Spitzgartner, dans le récit de Morand, fait débuter sa narration trente ans plus tôt: "C'était il y a trente ans et j'arrivais ici au sortir de l'Inspection des Finances» (Morand, 1984, p. 41). L'auteur ne remonte pas jusqu'à l'enfance de son personnage, mais jusqu'aux débuts de sa vie adulte. Psyché commence pour sa part par relater l'enfance de Sophonie, de manière à montrer les échelons gravis dans la maîtrise de son statut d'ogresse.

Par ailleurs, Hécate et ses chiens se composait de soixante-sept chapitres relativement succincts. L'ogresse en comporte 52 , tous brefs, voire très brefs (le $52^{\mathrm{e}}$ ne contient que sept mots). Un chapitre sur deux est en caractères réguliers, l'autre moitié en caractères italiques. La séquence en caractères normaux correspond aux événements de l'intrigue; celle en italiques renvoie à des apartés souvent précédés de la mention: "Aux dires de mes ancêtres ».

Si l'intrigue de L'ogresse renferme des éléments choquants - l'ingestion de fœtus risque d'en faire tressaillir plus d'un-, l'auteure née en 1955 n'a cependant rien de subversif. Après Cyclone (2008) et Zoélie du Saint-Esprit (2010), L'ogresse est son troisième roman pour adultes. Auparavant, elle avait été principalement active dans la littérature enfantine, avec des contes comme La tortue et l'araignée (2005) et Pourquoi Chien laisse-t-il pendre sa langue? (2008), de même qu'une série de fantasy en dix tomes, Gaïg. De toute évidence, Psyché ne saurait être soupçonnée, à l'instar de Wittkop, d'abhorrer les enfants.

\section{DÉVORATION ET SUBVERSION}

Même si, à l'encontre de Psyché, ni Morand ni Wittkop ne désignent clairement leurs héroïnes comme des ogresses $^{16}$, il n'en demeure pas moins que l'imaginaire sait de quoi il en retourne. Dans ses travaux sur la place de l'ogre, Jonathan F. Krell use d'ailleurs d'une acception large : l'ogre peut aussi bien désigner un personnage qu'une idée (la guerre, le nazisme, etc.). Personnage ou idée, un caractère vicieux s'affirme dans l'acte dévorateur (Krell, 2009, p. 17). Clotilde et la clientèle de Marguerite Paradis sont des ogres sexuels; Sophonie une ogresse cannibale... mais diffère-t-elle tant que ça des précédents? Elle énonce ainsi son statut: "L'ogritude totale dans une sexualité non moins totale» (Psyché, 2011, p. 66). Au moment d'ingérer son premier placenta, Sophonie éprouve, dans sa culotte, des ondes puissantes; elle a les jambes flageolantes, le sexe fourmillant (p. 71). Pour elle, une forme particulière d'extase naît « de la conjonction de l'humain dans [s] on sexe et dans [s]a bouche» (p. 66). Les plaisirs du palais et ceux du bas-ventre sont donc mis en étroite corrélation.

En raison de la violente voracité qui se déploie par le truchement de l'ogre, celuici a connu des avatars variés: politiques, sexuels et familiaux. Du point de vue politique, l'ogre, c'est tantôt Napoléon (que les royalistes avaient surnommé "l'ogre de Corse »), tantôt le III ${ }^{\mathrm{e}}$ Reich (qui fait d'Abel Tiffauges un ogre ${ }^{17}$ ). Du point de vue familial, il incarne le Mauvais Père, la Mauvaise Mère, qui tyrannise sa progéniture (ici, on pense au héros de Chessex) ou en abuse, la figure de l'ogre familial se superposant à celle de l'ogre sexuel dans le thème de l'inceste. Psyché a exploré cette dernière piste: un tableau «En guise d'arbre généalogique...»(p. 116-117) révèle les nombreuses ramifications incestueuses que comprend la lignée d'Euzèbe le Cannibale dont est issue la narratrice.

Les récits de Morand et de Wittkop développent le point de vue de l'ogre sexuel, plus particulièrement de l'ogresse, à travers deux femmes qui choisissent «d'ignorer l'instinct femelle» (Garcin, 2009, p. 43). Clotilde et Marguerite incarnent toutes deux des femmes qui, à un point ou à un autre de leur développement en tant qu'adultes, ont stagné au statut de filles (au sens filial). Ni belle ni laide (Morand, 1984, p. 44), Clotilde présente une grâce charmante qui doit beaucoup à son allure de femme-enfant, et fait preuve, dans son existence quotidienne, d'une insouciance toute juvénile: «Ce qu'il faut, reprit Clotilde après un silence, c'est se laisser vivre» (p. 45). Son mari, attaché politique, «s'attardait en Sibérie» (p. 47), 
la laissant libre de vaquer aux activités de son choix en «ce royaume du vide» (p. 46).

Ce qui surprend de prime abord, au sujet de Marguerite, dans La marchande d'enfants, c'est la hargne et l'absence de scrupules qu'elle manifeste à l'égard des enfants: "Les enfants étant des créatures si odieusement parasitaires et importunes, la Nature n'a pu assurer leur conservation qu'en opposant à un cannibalisme par trop justifié le verrou de l'amour parental» (Wittkop, 2003, p. 82). Sa position de femme jamais parvenue au degré de maturité (tout symbolique) de mère se confirme lorsque, faisant allusion à son enfance horrible, elle explique qu'elle n'a jamais voulu devenir maquerelle, mais qu'elle en vint «à une profession utile certes à l'humanité mais exécrable en elle-même et que chaque jour [elle] abomine davantage» (p. 141). Marguerite se venge de son enfance "abominable» (p. 139) en faisant le malheur des enfants qui croisent son chemin. Elle n'a guère le souci de protéger sa «marchandise », dont le sort consiste généralement à périr à l'issue du manège érotique dont sont victimes ces enfants (c'est d'ailleurs l'un des aspects les plus choquants du livre). Marguerite est une ogresse, mais l'époque en est une également. Ce n'est certainement pas un hasard si Wittkop a situé son intrigue en pleine tourmente révolutionnaire. Davantage qu'un simple élément de l'arrière-plan, la Révolution fait partie prenante du récit et il y aurait lieu, assurément, de poursuivre l'analyse dans cette voie.

En ce qui concerne le texte de Morand, un autre aspect (que je me contente de mentionner, même s'il appellerait un commentaire étendu) confirme sa valeur de réinterprétation du mythe de l'ogre: le fait que l'auteur y file la métaphore de l'appétit ogresque, avec des termes récurrents relatifs à la dévoration, à l'avidité et à la buccalité.

Qu'en est-il, dès lors, de la subversion? Chez Morand, nous l'avons vu, elle relève avant tout du biographique. Toute audacieuse que soit la thématique d'Hécate et ses chiens, Morand n'a rien d'un auteur scandaleux. Ce roman de 1954 est d'ailleurs l'une de ses rares excursions dans les eaux vraiment troubles du désir amoureux. L'écrivain d'après-guerre, en revanche, n'est pas au-dessus de tout soupçon. Lui qui avait été l'ami de Pierre Laval, il n'a certes jamais cautionné les politiques du gouvernement de Vichy, mais il fit l'erreur de ne pas les condamner non plus. Aussi a-t-il longtemps passé pour un auteur peu fréquentable, comme le rappelle Michel Collomb en ouverture de son essai, Paul Morand. Petits certificats de vie (p. 1).
En ce qui touche Wittkop, la subversion est omniprésente chez elle. J'y ai fait allusion précédemment au sujet du controversé roman Le nécrophile publié en 1972 (et réédité à deux reprises depuis ${ }^{18}$ ). Gabrielle Wittkop a laissé une œuvre d'une tonalité macabre, sensuelle, amorale et stylistiquement rigoureuse. "Je coquette sur deux choses : la langue et mon âge », affirmait l'octogénaire à Garcin (2009, p. 43). Son œuvre doit beaucoup à Sade. À cet égard, les discrètes apparitions du «divin marquis» dans La marchande d'enfants (il fait partie du cercle de connaissances de Marguerite) ne sont pas nécessaires pour nous en convaincre. Wittkop est une auteure sadienne, non seulement en raison de la grande hardiesse de ses propos en matière de «copulation », de "pédication » et autres divertissements libertins, mais aussi (et surtout) du point de vue axiologique. Elle partage et assume la philosophie de Sade. On sait que l'érotisme servait chez lui «de piment et d'introduction à ses idées sur la nature de l'homme en proie aux forces irrépressibles de la Nature nue », qu'il a été l'un des premiers «à avoir osé affirmer que l'injustice est dans la nature» (Sade, 1999, 4 ${ }^{\mathrm{e}}$ de couverture). Dans La marchande d'enfants, la Nature est foncièrement mauvaise. Les plus futés ou les plus roués en prennent acte et s'en accommodent autant que faire se peut. Certes, l'aristocratie décadente qui compose l'essentiel de la clientèle de Marguerite touche son point d'orgue en ce tournant des années 1790. Deux options surtout s'offrent à eux : la guillotine... ou l'exil. La maquerelle est avertie par une lettre, reçue d'un «sitoyen», que le vent s'apprête à tourner :

on nobserve que vous faite pratiqué la lucsure et la déboche et que vous la pratiqué vous même avèque les enfant du peuple souverin. On sai que vous éte une amie des aristocrate et autres si devant, on vous a vu séduire de ces enfants pour leur plus grande perdicion. On né au courant de vos ajissemens et les bon patriote ajiron en conséquensse car biento le moment est avenir.

A bon nentendeur salut (Wittkop,

2003, p. 128).

En langage clair, les jours de l'ogresse sont comptés. Le "peuple souverain» ne la laissera plus lui ravir ses enfants.

Or, on remarquera deux particularités ici. D'abord, si l'ogre se reconnaît à la violence de son activité dévoratrice, les insurgés - de «la foule agitée, qui [...] promène parfois des lambeaux de chair au bout des piques» (p. 139) au «tribunal révolutionnaire », qui «siège jour et nuit» (p. 141) - se qualifient eux aussi à porter le titre d'ogres. En fait, par leur association de la violence et de la licence extrêmes ${ }^{19}$, les années de la Révolution furent une période ogresque (celle, de surcroît, qui conduira à l'émergence de «l'ogre corse»).

D'autre part, les scènes évoquant «la luxure et la débauche» que cautionne Marguerite, y participant à l'occasion, poussent si loin l'opprobre qu'on pourrait s'attendre à ce que l'odieuse maquerelle soit punie, ou que ses non moins odieux clients finissent en subissant les foudres meurtrières du peuple souverain. La littérature, même dans la tradition réaliste (pensons au suicide d'Emma Bovary), nous a habitués à un minimum de moralité. Dans Les liaisons dangereuses, Valmont et Mme de Merteuil subissent les contrecoups de leur libertinage immodéré: le premier meurt en duel, la seconde perd la face (intimement et socialement). Or chez Wittkop, la morale ne vient rien sanctionner du tout. On perd la trace de Marguerite à la fin du roman. Certains bruits la prétendent morte; d'autres, en fuite. Au lecteur d'en déduire ce qu'il voudra. Ce qui n'échappera à personne, cependant, c'est que les monstres (la maquerelle et sa clientèle) auront eu la voie libre pour faire prospérer leurs vices. Les enfants y demeurent des victimes mal ou peu protégées.

Spitzgartner, dans Hécate et ses chiens, n'est pas davantage puni de ses inconduites. Se lancer à la recherche des plaisirs nocturnes de sa maîtresse l'a fait plonger dans la dépravation la plus condamnable. Il exploite une lacune pénale (la loi du pays ne reconnaît pas les mineurs). Il suscite toutefois l'embarras autour de lui. Les clients commencent à déserter sa banque. Il en est quitte pour un transfert. On a vu sanction plus sévère.

Dans L'ogresse non plus, la justice ne pèse pas lourd dans la balance. Sophonie ne fera guère les frais de son répréhensible "don». Au contraire, celui-ci a tout loisir de s'épanouir, car on l'a vu, L'ogresse relate l'évolution de la narratrice dans son « ogritude». Son appétit s'affirme et se raffine à la fois: "Je suis devenue plus vorace, certes, mais aussi plus gourmande, plus gastronome» (Psyché, 2011, p. 85). Tout comme l'ogresse végétarienne imaginée par Fleutiaux, cette ogresse fine bouche a de quoi frapper l'imagination. En même temps, Sophonie ne peut pas s'adonner tout à fait librement à son vice. Elle doit prendre diverses précautions, histoire de ne pas se mettre à dos la population qui fait mine de ne rien voir: "Personne n'a jamais rien dit, les gens étaient trop lâches pour intervenir, mais ils n'ignoraient rien de ce qui se passait» (p. 102). Son «instinct de chasseresse» (p. 86) doit s'accompagner de circonspection: «La vie en 
société est parfois dangereuse, il faut faire très attention à soi et surveiller sa façon d'agir» (p. 87). Aussi Sophonie a-t-elle mis au point tout un manège pour s'approvisionner. Elle a « appris à surveiller le cimetière» (p. 98). Lors d'enterrements, elle attend que la foule se dissipe pour assouvir sa faim. Parfois, si l'attente a été trop longue, la viande est devenue immangeable, ce qui déplaît à Sophonie: "Je l'ai dit, je ne suis pas un charognard, et festoyer sur des morts faisandés ne m'attire pas plus que ça» (p. 99). Tout comme Spitzgartner qui profitait d'une lacune pénale, Sophonie sait que la loi peut jouer en sa faveur: «La loi veut qu'on ne dérange pas un vivant qui travaille sur un mort, donc j'ai la paix » (p. 99). On effleure dès lors la dimension de critique sociale inhérente au mythe de l'ogre. "Dans quel monde maudit mange-t-on les timanmay? » se demande Sophonie (p. 64). Sophonie, tout comme Marguerite Paradis, a conscience qu'il y a quelque chose de pourri dans le royaume des hommes.

\section{APPROCHES DE L'ABJECTION}

À quelques décennies d'intervalle, Hécate et ses chiens, La marchande d'enfants et L'ogresse revisitent la figure de l'ogre en induisant, à travers elle, un processus de subversion mythologique, qui permet de définir une «approche de l'abjection». Leurs textes nous plongent dans le registre du scabreux et de l'insoutenable.

Or, cette dynamique de la subversion n'a rien d'improvisé. Elle donne lieu, dans les trois cas, à de savants dispositifs narratifs, surtout pour Morand: son usage de la suggestion et de l'ellipse fait d'Hécate et ses chiens un texte à relire à l'envi, ne fût-ce que pour apprécier le doigté de l'auteur dans sa sophistication de l'horreur.

La crudité du propos chez Wittkop et l'absence totale de compassion à l'égard des petites victimes - toutes désignées par des surnoms:

Verte-et-Bleue,

Cul mâché,

Martyrette et Guenillette,

Dorentin l'Aveuglé,

Lune rouge,

Sanguillon, Larmillon et Foutrillon,

Neige mouchetée,

Prince-des-Pleurs,

L'Enfant-Jésus,

Déchirure,

L'Empourprée,

Couronne-de-Douleur,

Tremblette,

Pâle Soyeuse,

Hurlante (Wittkop, 2003, p. 28).

- ce propos cru et cette intransigeance, dis-je, ne devraient pas faire oublier l'ampleur du travail du style accompli par Gabrielle Wittkop. Elle s'est réapproprié l'esprit du XVIII ${ }^{e}$ siècle et a poussé l'audace dans ses derniers retranchements. Pour elle, l'inexploré n'est pas de l'inexplorable; elle l'avait démontré avec Le nécrophile et elle en fait à nouveau la preuve. "On peut écrire sur tout, soulignait-elle, mais il faut savoir comment » (Blin, 2003).

La même affirmation peut s'appliquer au livre de Dynah Psyché. Du strict point de vue des thèmes abordés, L'ogresse n'offre rien de très recommandable: cannibalisme, meurtre, nécrophilie, inceste... Pourtant, la solidité du dispositif narratif nous évite de sentir qu'il s'agit d'une lecture pour déséquilibrés. Pour les besoins de mon propos, je m'en suis tenu à certains aspects. Bien d'autres auraient pu retenir notre attention. Je pense notamment aux qualités musicales de la prose de Psyché. Le chapitre 29, par exemple, est bâti sur une série d'anaphores:

Le don aime la mer et le don invente

le nager comme un poisson.

Le don aime le vent et le don invente

le bruissement des ailes invisibles.

Le don aime la musique et le don

invente un nouveau jouer.

Le don aime la boisson et le don

invente la grande soif.

[...]

Le don aime le voyage et le don

invente le pas infini.

Le don aime le temps et le don

invente la mémoire totale.

Le don aime le chat et invente le voir dans la nuit noire.

Le don aime les enfants et le don invente la bonne chère.

Le don aime les langues et le don joue avec les mots.

Mais toujours le protègement du don protège le possessionneur du don (p. 74-75).

Cette qualité de la prose me paraît nécessaire pour que le charme (tout négatif soit-il) opère.

La dimension psychologique a elle aussi été étoffée. Sophonie est une ogresse qui prend conscience d'elle-même: "J'ai vite découvert que posséder la compréhension, en plus de la sensation, ça amplifie le plaisir» (p. 85). Mais sommes-nous réellement dans les eaux troubles du plaisir ou ne s'agit-il pas, en fin de compte, d'un cercle vicieux qui s'apprête à se refermer sur le «possessionneur du don»? L'ogresse se conclut sur deux perspectives inquiétantes: l'«infantiphagie» (après avoir mangé le père de son enfant, la narratrice, enceinte, se prépare à manger ce dernier: "Plus tu restes là, plus tu engraisses, meilleur tu seras» [p. 113]) et l'autophagie («tu te mangeras toi-même» [p. 114]). Il y aurait lieu de relire L'ogresse à la lumière de la psychanalyse.

En fait, quoique l'œuvre de Psyché soit encore toute récente en comparaison de celles de Morand et de Wittkop, on peut considérer L'ogresse comme un texte qu'on prendra plaisir à relire. Comme quoi, un travail du style, s'il est bien mené, donne (pratiquement) le champ libre aux romanciers. On n'a qu'à penser au cas de L.-F. Céline.

Morand, quant à lui, ne donne qu'en apparence davantage dans la dentelle, car le fond de son récit est lui aussi odieux. Or-comme je l'ai montré ailleurs ${ }^{20}-$, il a choisi de situer son sujet en contrepoint de la psychanalyse afin de suivre le filon de l'obsession amoureuse. Spitzgartner a toutes les allures du narrateur non fiable (unreliable narrator), car l'ogresse qu'il nous dépeint trouvera le moyen de lui faire savoir, lorsqu'ils se reverront brièvement à New York en 1942: "vous étiez terriblement vicieux» (Morand, 1984, p. 158).

Et nous, qui sortons de cette lecture avec les mêmes sentiments partagés que lorsque nous sortons de Lautréamont, de Bataille ou du Nabokov de Lolita, nous sommes bien forcés d'admettre qu'elle n'a pas tort.

\section{Bibliographie}

BLIN, R. (2003). "La Marchande d'enfants de Gabrielle Wittop », Le Matricule des Anges, $\mathrm{n}^{\circ}$ 046, en ligne, <http://www.lmda. net/din/tit_lmda.php?Id=17285>, consulté le 2011-05-25.

BOULOUMIÉ, A. (1992). «The ogre in literature ", dans P. BRUNEL (dir.), Companion to Literary Myths, Heroes, and Archetypes, Londres, Routledge, p. 912-924.

BRUCKNER, P. (1998). Les ogres anonymes suivi de L'effaceur, Paris, Grasset.

BURGESS, A. (1972). L'orange mécanique, trad. de Georges Belmont et Hortense Chabrier, Paris, Livre de poche.

CHESSEX, J. (2009). L'ogre, Paris, Grasset, coll. «Les cahiers rouges».

COLLOMB, M. (2007). Paul Morand, Petits certificats de vie, Paris, Hermann.

FLEUTIAUX, P. (2010). Métamorphoses de la reine, Paris, Gallimard, coll. "Folio».

GARCIN, J. (2009). "La vieille dame indigne", dans Les livres ont un visage, Paris, Mercure de France, p. 36-45.

KRELL, J.F. (1994). «Quelques ogres dans la littérature française contemporaine: Tournier, Chessex, Pennac », dans M. ZUPANCIC (dir.), Mythes dans la littérature contemporaine d'expression française. Actes du colloque tenu à l'Université d'Ottawa du 24 au 26 mars 1994, Ottawa, Le Nordir, p. 247-255.

KRELL, J.F. (1998). "L'ogre réécrit, l'ogre réhabilité », dans Cahiers de l'imaginaire 16: Esthétique, littérature et modernité, Paris, L'Harmattan, p. 15-20. 
KRELL, J.F. (2004). "The dark side of Aphrodite: the ogress in the fiction of Michel Tournier and Jacques Chessex », dans M. ZUPANCIC (dir.), Hermes and Aphrodite Encounters, Birmingham (Alabama), Summa Publications, p. 99-108.

KRELL, J.F. (2005). «Les résurgences de l'ogre dans la littérature contemporaine: entretiens avec Michel Tournier et Pascal Brucker », dans Héroïsation et questionnement identitaire en Occident: Héroïsation/ antihérö̈sation-civilisation/ barbarie. Cahiers électroniques de l'imaginaire 2, p. 155-166, en ligne, <http://www.e-montaigne.com/>, consulté le 2011-05-25.

KRELL, J.F. (2009). The Ogre's Progress, Images of the Ogre in Modern and Contemporary French Fiction, Newark, University of Delaware Press.

KRISTEVA, J. (2007). Pouvoirs de l'horreur, Paris, Seuil, coll. «Points Essais».

LAUTRÉAMONT (1963). Les chants de Maldoror, Paris, Livre de poche.

MORAND, P. (1984). Hécate et ses chiens, éd. Marc Dambre, Paris, Flammarion, coll. «GF».

PENNAC, D. (2003). Au bonheur des ogres, Paris, Gallimard, coll. «Folio».

PERRAULT, C. (2004). Contes, Paris, Gallimard, coll. «Folio».

PSYCHÉ, D. (2011). L'ogresse, Montréal, Coups de tête.

RAUCH, A. (2006). Histoire du premier sexe: De la Révolution à nos jours, Paris, Hachette, coll. «Pluriel Sociologie».

SADE, D.A.F. (DE) (1999). L'ogre Minski, Paris, Le Mercure de France, coll. «Le petit Mercure».

TOURNIER, M. (1986). Gilles et Jeanne, Paris, Gallimard, coll. «Folio ».

TOURNIER, M. (1996). Le roi des aulnes, Paris, Gallimard, coll. «Folio».

WITTKOP, G. (2003). La marchande d'enfants, Paris, Verticales.

\section{Notes}

1. Une première version de cette étude a été présentée sous forme de conférence à l'occasion du colloque international "Mythe et subversion dans le roman contemporain» (Université Complutense, Madrid, 9-11 mars 2011).

2. Il s'agit, dans l'ordre, de: Émile Zola, Le ventre de Paris; Michel Tournier, Le Roi des Aulnes et «Les suaires de Véronique» dans $L e c o q$ de bruyère; Daniel Zimmermann, Le dixième cercle: l'anus du monde; Pierrette Fleutiaux, "La femme de l'Ogre », dans Métamorphoses de la reine; Sylvie Germain, Jours de colère et L'enfant méduse; Muriel Cerf, Ogres et autres contes et Le verrou; Jacques Chessex, L'ogre; Daniel Pennac, Au bonheur des ogres; Pierre Péju, Le rire de l'ogre. D'autres références sont citées en bibliographie (p. 156-162).

3. Charles Perrault (1628-1703), auteur des célèbres Contes de ma mère l'oye (1697), dont font notamment partie « La belle au bois dormant», «Le petit chaperon rouge», «Le maître chat ou le chat botté », "Cendrillon ou la petite pantoufle de verre» et «Le petit Poucet».

4. De «L'affaire Lafargue», dont s'est inspiré Stendhal dans Le rouge et le noir (1830), à «L'affaire Dominici » ou "L'affaire Romand », qui ont respectivement donné lieu à $L a$ tragédie de Lurs de Jean Meckert et L'adversaire d'Emmanuel Carrère, la liste est longue des romanciers qui ont puisé leur inspiration dans des faits divers. On pourra lire à ce propos l'article d'Hubert Artus, «Le fait divers reste de la chair à fiction", en ligne, $<$ http://www.rue89.com/cabinet-de-lecture/ le-fait-divers-reste-de-la-chair-a-fiction $>$, Rue89, 2007-07-23, consulté le 2011-05-25.

5. L'usage de ce terme fait référence au roman dystopique d'Anthony Burgess, L'orange mécanique (A Clockwork Orange). C'est Alex, le narrateur, qui se sert de l'expression: «faire les ultra-violents » (Burgess, 1972, p. 10).

6. Prenons un exemple récent. Dans son adaptation du roman de Tatiana de Rosnay, Elle s'appelait Sarah, le réalisateur Gilles PaquetBrenner a choisi d'utiliser la technique hitchcockienne du reaction shot lors d'une scène où les personnages font la découverte du cadavre d'un enfant qui s'était caché dans un placard. On ne voit rien du petit corps, mais la réaction horrifiée des personnages capture toute l'émotion que ce revirement dramatique devait inspirer.

7. «Il y a, dans l'abjection, une de ces violentes et obscures révoltes de l'être contre ce qui le menace et qui lui paraît venir d'un dehors ou d'un dedans exorbitant, jeté à côté du possible, du tolérable, du pensable. C'est là, tout près mais inassimilable. Ça sollicite, inquiète, fascine le désir qui pourtant ne se laisse pas séduire. Apeuré, il se détourne. Écœuré, il rejette. Un absolu le protège de l'opprobre, il en est fier, il y tient. Mais en même temps, quand même, cet élan, ce spasme, ce saut, est attiré vers un ailleurs aussi tentant que condamné. Inlassablement, comme un boomerang indomptable, un pôle d'appel et de répulsion met celui qui en est habité littéralement hors de lui» (Kristeva, 2007, p. 9).

8. "The most terrible ogre is the one who practices "infantiphagia": eating his own children» (Michel Tournier, entretien avec J.F. Krell, Choisel, France, 19 mai 2002; Krell, 2009, p. 136.)

9. Voir le début du premier chapitre: «S'il [Euzèbe] n'était pas allé prendre l'enfant d'Euphémie pour le manger, les choses seraient différentes aujourd'hui. L'enfant était mort, oui, mort-né, même, mais de là à consommer de l'humain, il y avait une limite à ne pas franchir. [...] Il a prétendu qu'il croyait que c'était le sien! Donc, qu'il pouvait le manger!» (Psyché, 2011, p. 5.)

10. Jérôme Garcin, qui a rencontré l'auteure en décembre 2000, rapporte que dans la chaîne de ses dégoûts, la "haine des enfants » est prioritaire: "J'adore les animaux. Ce n'est pas comme les gosses. Ah, les saletés. Quand je les vois jouer sur la pelouse, je les chasse! [...] Dès mon plus jeune âge, j'ai détesté les gamins » (Garcin, 2009, p. 36-37).
11.Pour des variations sur le mythe d'Hécate, se reporter aux œuvres suivantes: Jouve, Pierre Jean. Aventure de Catherine Crachat I. Hécate. Paris: Gallimard, 1991. Folio; Gadenne, Paul. L'invitation chez les Stirl. Paris : Gallimard, 1982. Folio; Noël, Bernard. Le château de Cène suivi de Le château de Hors. L'outrage aux mots. La pornographie. Paris: Gallimard, 2005. L'imaginaire; Nizan, Paul-Yves. Complainte du carabin qui disséqua sa petite amie en fumant deux paquets de maryland suivi de Hécate ou la méprise sentimentale. Le Revest-les-Eaux: Éditions Spectres Familiers, 1982.

12. Selon le site <http://www.1789-1815.com/ cal_04_3.htm>, consulté le 2011-05-25.

13. Citation originale: «Plût au ciel que le lecteur, enhardi et devenu momentanément féroce comme ce qu'il lit, trouve, sans se désorienter, son chemin abrupt et sauvage, à travers les marécages désolés de ces pages sombres et pleines de poison; car, à moins qu'il n'apporte dans sa lecture une logique rigoureuse et une tension d'esprit égale au moins à sa défiance, les émanations mortelles de ce livre imbiberont son âme comme l'eau le sucre. Il n'est pas bon que tout le monde lise les pages qui vont suivre; quelques-uns seuls savoureront ce fruit amer sans danger» (Lautréamont, 1963, p. 41).

14. En plus de néologismes issus de la déformation de termes existants, comme possessionneur (p. 14) et portateur (p. 52), Psyché use de mots exotiques comme timanway (" enfant», p. 43) ou bòbò («putain», p. 72).

15. Voire trois, puisque Psyché recourt elle aussi aux motifs des chiens et de la chasse.

16. Pour l'anecdote, on notera que la marchande d'enfants se sert une fois du terme " ogresse», en appliquant à la vieille femme auprès de qui elle «négocie l'achat» d'un jeune hermaphrodite, que Marguerite surnommera Tirésias et auquel elle s'attachera particulièrement (Wittkop, 2003, p. 110). Morand, pour sa part, use parcimonieusement du terme. Je n'ai relevé qu'une occurrence: «Jouait-elle? Et à quoi ? À la maîtresse d'école ou à la princesse de Bagdad, à l'héroïne de roman ou à l'ogresse? Ou, simplement, était-elle restée puérile, sous l'enveloppe d'une grande personne? » (Morand, 1984, p. 138-139.)

17. Un ogre ambivalent, car Tournier a aussi voulu lier son personnage à la figure de saint Christophe.

18. Aux Éditions La Musardine en 1998 et Verticales en 2001. Notons qu'une traduction anglaise vient de paraître aux Éditions ECW, The Necrophiliac, dans une traduction de Don Bapst.

19. Dans son Histoire du premier sexe, André Rauch a bien montré la prolifération d'écrits obscènes à l'époque. Voir le premier chapitre: "Le nouveau corps des citoyens» (p. 23-49).

20.P. Bergeron, «Fictions d'Hécate, de Jouve à Morand», dans BERGERON, P. et M. CARRIÈRE (dir.), Les réécrivains: enjeux transtextuels dans la littérature moderne d'expression française, Berne, Peter Lang, coll. "Littératures de langue française » (sous presse). 